\title{
Impact of Channel Estimation Errors on SC-FDE Systems
}

\author{
Nuno Souto, Rui Dinis, Member, IEEE, and João Carlos Silva
}

\begin{abstract}
Single carrier transmissions with frequency domain equalization (SC-FDE) have gained widespread use in emergent broadband wireless systems becoming an attractive alternative to popular Orthogonal Frequency Division Multiplexing (OFDM) schemes, particularly at the uplink. Since coherent receivers are usually employed with SC-FDE, accurate channel estimates are required so as to avoid substantial performance degradation. Several channel estimation strategies have been proposed for SC-FDE, but a thoroughly evaluation of the degradation caused by channel estimation errors and a comparison against OFDM is still lacking. In this paper we study the impact of imperfect channel knowledge on SC transmission with focus on the linear frequency domain equalizer (FDE) and on the Iterative Block Decision Feedback Equalizer (IB-DFE). We propose a modified IB-DFE which incorporates knowledge of the channel estimation error model and show that its performance becomes more robust against the presence of strong error components in the channel estimates. We also evaluate, analytically and through simulations, the degradation caused by imperfect channel estimation in SC-FDE and compare it against OFDM schemes (Orthogonal Frequency Division Multiplexing). It is shown that the channel estimation requirements for SC-FDE are higher than for OFDM unless a channel estimation error aware receiver is employed.
\end{abstract}

Index Terms-Channel estimation, frequency-domain equalization, iterative equalization, OFDM, SC-FDE.

\section{INTRODUCTION}

$\mathbf{T}$ HE ever increasing demand of high transmission rates in broadband wireless systems poses major design challenges specially due to the severe time dispersion occurring in the radio channel. Emergent radio systems often resort to block transmission techniques with frequency domain equalization (FDE) methods in order to sustain the envisioned high data rates. Due to its implementation simplicity both in the transmitter and receiver, OFDM schemes [1] are the most popular and have been incorporated into several communications standards, as for example Digital Video Broadcast

Manuscript received January 16, 2013; revised July 19 and October 8, 2013 The associate editor coordinating the review of this paper and approving it for publication was A. Tonello.

$\mathrm{N}$. Souto is with the ISCTE-University Institute of Lisbon and Instituto de Telecomunicações, 1649-026 Lisboa, Portugal (e-mail: nuno.souto@lx.it.pt).

R. Dinis is with the Instituto de Telecomunicações and the Faculty of Science and Technology-UNL, Monte da Caparica Lisboa, Portugal, 2829. 516 Caparica, Portugal (e-mail: rdinis@fct.unl.pt).

J. C. Silva is with the ISCTE-University Institute of Lisbon and Instituto de Telecomunicações, 1649-026 Lisboa, Portugal (e-mail: joao.carlos.silva@1x.it.pt).

This work was partially supported by the FCT - Fundação para a Ciência e Tecnologia (projects ADIN - Advanced PHY/MAC Design for Infrastructure-less Networks PTDC/EEI-TEL/2990/2012, LTE-Advanced Enhancements using Femtocells PTDC/EEA-TEL/120666/2010 and PEstOE/EEI/LA0008/2013).

This paper was presented in part at the IEEE 77th Vehicular Technology Conference Spring, Dresden, Germany, June 2013.

Digital Object Identifier 10.1109/TCOMM.2013.121613.130046
(DVB) [2], Long Term Evolution (LTE) [3] and WiMax [4]. However, OFDM has some weaknesses and in particular its large peak to average power ratio (PAPR) hinders its use in the uplink connection of mobile communication systems. For this case single carrier block transmissions with frequency domain equalization (SC-FDE) [5], [6] is widely accepted as a more attractive solution since it allows reliable transmission in strong inter-symbol interference (ISI) inducing channels while avoiding the large PAPR associated to OFDM signals based on the same constellations. SC schemes have already been incorporated into several wireless standards, such as the adoption of single carrier frequency division multiple access (SC-FDMA) at the uplink of LTE [3]. Although many comparisons have been made in the literature between OFDM and SC schemes [7]-[10], a thoroughly comparison taking into account the impact of imperfect channel estimation on both systems is still lacking.

Conventional SC-FDE schemes employ a linear FDE which has low implementation complexity. However, its performance falls short from the matched filter bound (MFB), which means that there is significant room for performance improvements [11]. To improve the performance and reduce the gap relatively to the MFB we should replace the conventional linear FDE by a nonlinear equalizer. The most popular nonlinear equalizers implemented in the time domain are decision feedback equalizers (DFE), but their complexities can become quite high in severely time dispersive channels; moreover, conventional DFE implementations can suffer error propagation problems [12]. To overcome these issues, a promising DFE where the feedforward and feedback parts are implemented in the frequency domain was proposed in [13]. This technique, denoted IB-DFE (Iterative Block Decision Feedback Equalizer), was later extended to a wide range of scenarios with different complexity/performance tradeoffs [12]-[17]. It was shown that the IB-DFE is able to achieve performances close to MFB in rich multipath propagation channels with affordable complexity [12], [18], [19], as long as accurate channel estimates are available. Even though the channel estimation problem has been previously studied in the literature, the main concern has been on proposing different pilot transmission strategies [20]-[22] and reliable estimation algorithms [23], [24]

In this paper we study the impact of channel estimation errors on the performance of SC-FDE transmission, focusing in particular on the use of a linear DFE and a IB-DFE. As most equalizers [12], the original IB-DFE was designed assuming perfect channel knowledge [13], [14] and, therefore, even if the performance of a channel estimator is known, namely the estimator' bias and error variance, this knowledge is not used 
by the equalizer. In this paper we propose a new modification on the IB-DFE in order to take into account the channel estimation error characteristics. Furthermore, novel analytical BER expressions are derived for evaluating the performance of the linear FDE and the IB-DFE in the presence of imperfect channel estimation allowing a comparison against uncoded OFDM. This comparison is also extended to the coded case through simulations.

The paper is organized as follows. Section II describes the system model and the proposed modified IB-DFE which incorporates knowledge of the channel estimation error variance. Section III derives BER expressions for the linear FDE and also for an ideal IB-DFE, both considering imperfect channel estimation. Section IV presents and analyzes some numerical results for SC-FDE with imperfect channel estimation and compares it with OFDM. Finally the conclusions are given in Section V.

\section{IB-DFE RECEIVER WITH IMPERFECT CHANNEL ESTIMATION}

In this section we will extend the approach employed in [14] and [16] in order to obtain a more robust IB-DFE in the presence of channel estimation errors.

\section{A. System Model}

Let us consider a single carrier transmission where each set of $N$ modulated symbols, $s_{n},(n=1, \ldots, N)$, is grouped into a block and to which a suitable cyclic prefix (CP) is appended. The resulting block is then transmitted through the channel. For reliable detection of the transmitted symbols the proposed IB-DFE receiver operates in the frequency domain, as shown in Fig. 1. In this figure it is assumed that the signal has been previously filtered, down converted, sampled and the $\mathrm{CP}$ has been removed from each block. The resulting time domain samples constituting a size- $N$ block, $y_{n}(n=1, \ldots, N)$, are converted to the frequency domain through an $N$-point DFT (Discrete Fourier Transform) resulting the following expression for the frequency domain samples

$$
Y_{k}=H_{k} S_{k}+N_{k}
$$

where $k=1, \ldots, N$ is the subcarrier index, $H_{k}$ are the channel frequency response coefficients, $S_{k}$ are the DFT samples of the modulated symbols $s_{n}$ and $N_{k}$ corresponds to the noise frequency domain samples. It is assumed that both Sk and $\mathrm{Nk}$ are zero mean complex random variables with variances $P_{S}=E\left[\left|S_{k}\right|^{2}\right]$ and $P_{N}=E\left[\left|N_{k}\right|^{2}\right]=N \cdot N_{0}$ ( $N_{0}$ is the noise power spectral density).

The channel frequency response coefficients, $H_{k}$, are related to the channel impulse response according to

$$
H_{k}=D F T\left\{h_{n}\right\}=\sum_{n=1}^{L} h_{n} \exp \left(-j 2 \pi \frac{(k-1)(n-1)}{N}\right),
$$

where $L$ is the number of resolvable propagation paths and $h_{n}$ are the respective impulse response coefficients, modeled as independent zero-mean complex Gaussian random variables.

Regarding the channel estimates required for the equalization, we will assume the transmission of pilot symbols and

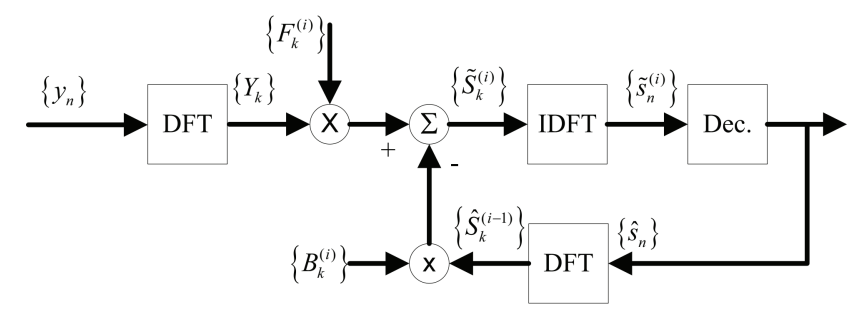

Fig. 1. IB-DFE receiver structure.

the use of an arbitrary linear channel estimator which can be represented as

$$
\widehat{\mathbf{H}}=\mathbf{F} \cdot \mathbf{Y},
$$

where $\widehat{\mathbf{H}}$ is a $N \times 1$ column vector whose elements $\widehat{H}_{k}$ are the channel estimates at subcarrier index $k, \mathbf{Y}$ is a $N \times 1$ column vector containing the frequency domain samples $Y_{k}$ and $\mathbf{F}$ represents the filter matrix. Note that expression (3) is general, in the sense that it can correspond to an estimator based on a block type or a comb type pilot arrangement [25].

Since the frequency domain samples $Y_{k}$ are zero-mean complex Gaussian distributed random variables, the channel estimates obtained using (3) will also be zero-mean Gaussian distributed. Therefore we can decompose as the sum of a scaled version of $\widehat{H}_{k}$ (a zero mean complex Gaussian variable) and a noise term $\varepsilon_{k}$, which is also a zero mean complex Gaussian variable with variance $P_{\varepsilon}=E\left[\left|\varepsilon_{k}\right|^{2}\right]$. Estimate $\widehat{H}_{k}$ can thus be written as

$$
\widehat{H}_{k}=a \cdot H_{k}+\varepsilon_{k},
$$

where $a$ is a complex value chosen so as to guarantee that $H_{k}$ and $\varepsilon_{k}$ are uncorrelated, i.e., $E\left[\varepsilon_{k} H_{k}^{*}\right]=0$. It is simple to verify that this condition is satisfied when

$$
a=\frac{E\left[\widehat{H}_{k} H_{k}^{*}\right]}{E\left[H_{k}\right]^{2}}
$$

Leading to

$$
P_{\varepsilon}=E\left[\left|\varepsilon_{k}\right|^{2}\right]=E\left[\left|\widehat{H}_{k}\right|^{2}\right]-a E\left[\left|\widehat{H}_{k}^{*} H_{k}\right|\right] .
$$

It is important to note that the channel estimation model defined in (4) is similar to the one used in [26] and is valid even if $H_{k}$ is not Gaussian (naturally, in this case $\varepsilon_{k}$ will follow a different distribution).

According to (5), $a$ and consequently $P_{\varepsilon}$, are constant for each $k$ but can differ for different subcarriers, depending on the channel estimator employed. However, for readability, in the remainder of this paper it will be assumed as implicit that both $a$ and $P_{\varepsilon}$ may depend on $k$, although no explicit index will be attached to these variables.

\section{B. Derivation of IB-DFE Coefficients}

Sequence $Y_{k}$ is processed iteratively by the IB-DFE generating estimates in the frequency domain. For each iteration $\mathrm{i}$ the estimates can be written as

$$
\widetilde{S}_{k}^{(i)}=F_{k}^{(i)} Y_{k}-B_{k}^{(i)} \widehat{S}_{k}^{(i-1)},
$$


where $F_{k}$ represents the feedforward coefficient, $B_{k}$ are the feedback coefficient and $\widehat{S}_{k}^{(i-1)}$ are the DFT samples of the estimated block from the previous iteration after the decision device. The feedforward and feedback coefficients are computed in order to minimize the mean squared error (MSE) between the estimated symbols and the transmitted symbols at the detection point of the receiver. In [13] and [14] expressions for $F_{k}$ and $B_{k}$ were derived assuming perfect channel knowledge. In the following we obtain expressions for the same coefficients considering imperfect channel estimation with known channel estimation error model.

Regarding the feedback samples $\widehat{S}_{k}^{(i-1)}$ required in (7), they are obtained from the soft decisions at the FDE output, i.e.,

$$
\widehat{S}_{k}^{(i)}=\sum_{n=0}^{N-1} \widehat{s}_{n}^{(i)} e^{-\frac{j 2 \pi k n}{N}}
$$

with

$$
\widehat{s}_{n}^{(i)}=E\left[s_{n} \mid \widetilde{s}_{n}^{(i)}\right]=\sum_{s \in \Lambda} s \cdot p\left(s_{n}=s \mid \widetilde{s}_{n}^{(i)}\right),
$$

where $p\left(s_{n}=s \mid \widetilde{s}_{n}^{(i)}\right)$ represents the probability of the correct symbol $s_{n}$ being $s$ when the equalizer ouput is $\widetilde{s}_{n}$ (see [27]). In the summation, $\Lambda$ represents the set of constellation symbols. It is possible to show that the equalizer output can be written as

$$
\widetilde{s}_{n}^{(i)}=s_{n}+\xi_{n}^{(i)}
$$

where $\xi_{n}^{(i)}$ represents noise and ISI. This term can be approximated as a zero mean complex Gaussian random variable [14] with variance $2 \sigma_{\xi}^{2_{(i)}}$ and, therefore, $p\left(s_{n}=s \mid \widetilde{s}_{n}^{(i)}\right)$ can be computed as

$$
p\left(s_{n}=s \mid \widetilde{s}_{n}^{(i)}\right)=K e^{-\frac{\left|s-\widetilde{s}_{n}^{(i)}\right|^{2}}{2 \sigma_{\xi}^{2_{(i)}}},}
$$

where $K$ is a normalizing constant. For the implementation of the proposed equalizer it is necessary to estimate $2 \sigma_{\xi}^{2_{(i)}}$ and $E\left[\widehat{S}_{k}^{(i)} S_{k}^{*}\right]$. The former one can be accomplished by using

$$
2 \sigma_{\xi}^{2(i)}=\frac{1}{N} \sum_{n=0}^{N-1}\left|\widehat{s}_{n}^{(i)}-\widetilde{s}_{n}^{(i)}\right|^{2}
$$

with $\widehat{s}_{n}^{(i)}$ representing the hard decision symbol, while $E\left[\widehat{S}_{k}^{(i)} S_{k}^{*}\right]$ can be estimated using the approach described in [14].

Let us define the second order moment for the feedback samples as $P_{\widehat{S}}=E\left[\left|S_{k}^{(i-1)}\right|^{2}\right]$. The aim is to find the coefficients $F_{k}$ and $B_{k}$ that minimize the MSE at the detection point conditioned on $\widehat{H}_{k}$. Using $(\cdot)^{*}$ for denoting the complex conjugate, we can express the MSE as (13)-(17) at the top of the next page.

These expressions were obtained taking into account that, according to (4), the received samples in the frequency domain (1) can also be written as

$$
Y_{k}=a^{-1} \widehat{H}_{k} S_{k}-a^{-1} \varepsilon_{k} S_{k}+N_{k} .
$$

The MSE expression (13) has to be minimized under the constraint that the feedback component of the IB-DFE does not remove the desired signal component [14], i.e.,

$$
\sum_{n=0}^{N-1} B_{k}^{(i)}=0
$$

We can then apply the Lagrange multipliers method through a minimization of the function

$$
\Psi\left(F_{k}^{(i)}, B_{k}^{(i)}, \lambda\right)=M S E+\operatorname{Real}\left\{\lambda^{*} \sum_{n=0}^{N-1} B_{k}^{(i)}\right\}
$$

which, being a real function with complex variables, can be accomplished using

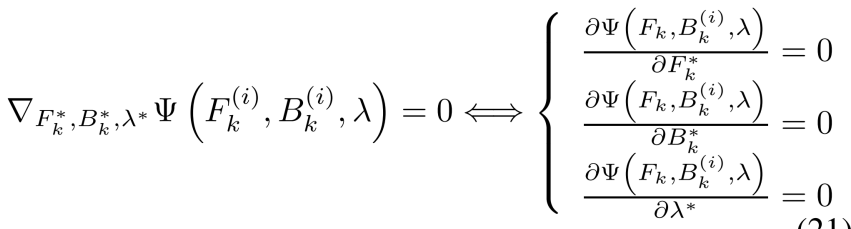

leading to (22) on the next page and

$$
B_{k}^{(i)}=\left(a^{-1} F_{k}^{(i)} \widehat{H}_{k}-1\right) \frac{E\left[S_{k} \widehat{S}_{k}^{(i-1) *} \mid \widehat{H}_{k}\right]}{P_{\widehat{S}}} .
$$

Parameter $\gamma$ is selected to ensure that (19) is fulfilled, i.e., we must have

$$
\frac{1}{N} \sum_{k=0}^{N-1} a^{-1} F_{k}^{(i)} \widehat{H}_{k}=1 .
$$

The main difference resulting from the incorporation of the knowledge of the variance of channel estimation error into this IB-DFE version compared with the one presented in [13] and [14] lies in the existence of the term $|a|^{-2} P_{S} P_{\varepsilon}$ in (22).

\section{Single Carrier Performance Analysis}

Following the derivation of the IB-DFE, in this section we address its performance analysis, starting with the case of the lower complexity linear FDE followed by the case of an ideal IB-DFE.

\section{A. Linear FDE}

Let us start by analyzing the behavior of the IB-DFE with only one iteration, which corresponds to a linear FDE optimized under the Minimum Mean Square Error (MMSE) criterion. For the linear FDE only the feedforward coefficients, $\mathrm{Fk}$, are required which can be obtained using

$$
F_{k}=\frac{\gamma\left(a^{-1}\right)^{*} \widehat{H}_{k}^{*}}{|a|^{-2}\left|\widehat{H}_{k}\right|^{2} P_{s}+|a|^{-2} P_{S} P_{\varepsilon}+P_{N}} .
$$

Taking into account that the output of the equalizer can be written as (10) and assuming a QPSK constellation with Gray mapping, it is easy to see that the bit error rate (BER) dependent on a channel realization can be computed with [28]

$$
P_{b} \approx Q\left(\sqrt{\frac{2 E_{b}}{M S E}}\right),
$$

